\title{
INDEX MAPS IN THE $K$-THEORY OF GRAPH ALGEBRAS
}

\author{
TOKE MEIER CARLSEN, SØREN EILERS, AND MARK TOMFORDE
}

\begin{abstract}
Let $C^{*}(E)$ be the graph $C^{*}$-algebra associated to a graph $E$ and let $J$ be a gauge-invariant ideal in $C^{*}(E)$. We compute the cyclic six-term exact sequence in $K$-theory associated to the extension

$$
0 \longrightarrow J \longrightarrow C^{*}(E) \longrightarrow C^{*}(E) / J \longrightarrow 0
$$

in terms of the adjacency matrix associated to $E$. The ordered sixterm exact sequence is a complete stable isomorphism invariant for several classes of graph $C^{*}$-algebras, for instance those containing a unique proper nontrivial ideal. Further, in many other cases, finite collections of such sequences comprise complete invariants.

Our results allow for explicit computation of the invariant, giving an exact sequence in terms of kernels and cokernels of matrices determined by the vertex matrix of $E$.
\end{abstract}

\section{INTRODUCTION}

The cyclic six-term exact sequence

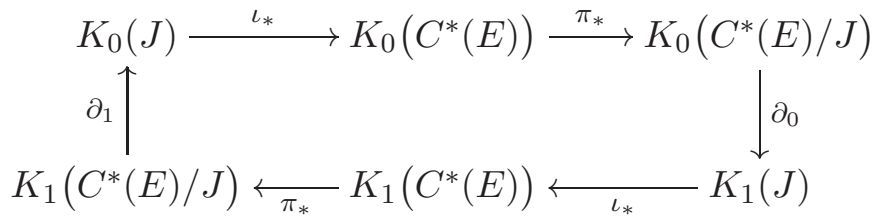

is a complete stable isomorphism invariant for a graph $C^{*}$-algebra $C^{*}(E)$ of real rank zero containing a proper nontrivial ideal $J$ when any of the following are satisfied

- $J$ is the unique proper nontrivial ideal of $C^{*}(E)$ ([7, Theorem 4.5]),

- $J$ is a smallest proper nontrivial ideal of $C^{*}(E)$, and $C^{*}(E) / J$ is $\mathrm{AF}$ ([6. Corollary 6.4]),

- $J$ is a largest proper nontrivial ideal of $C^{*}(E)$, and $J$ is $\mathrm{AF}(7$, Theorem 4.7]).

Date: October 25, 2018.

2000 Mathematics Subject Classification. 46L55.

Key words and phrases. graph $C^{*}$-algebras, classification, extensions, $K$-theory.

This research was supported by the NordForsk Research Network "Operator Algebras and Dynamics" (grant \#11580). The first named author was supported by the Research Council of Norway. The third author was supported by NSA Grant H98230-09-1-0036. 
In other cases (cf. [6]) a complete invariant may be obtained by combining several six-term exact sequences associated to $C^{*}(E)$ and its ideals.

It is therefore important to address how to compute sequences of the form in (1.1). In the existing literature it is shown that if $E$ is a row-finite graph with no sinks, then

$$
K_{0}\left(C^{*}(E)\right) \cong \operatorname{coker}\left(A^{t}-I\right) \text { and } K_{1}\left(C^{*}(E)\right) \cong \operatorname{ker}\left(A^{t}-I\right),
$$

where $A^{t}-I: \mathbb{Z}^{E^{0}} \rightarrow \mathbb{Z}^{E^{0}}$ is the linear map given by the transpose of the vertex matrix $A$ of $E$ minus the identity matrix $I$. This description of the $K_{0^{-}}$ group also includes a description of its order, and a similar computation exists when sinks and infinite emitters are allowed. Since gauge-invariant ideals of graph $C^{*}$-algebras and the corresponding quotients are naturally isomorphic to graph $C^{*}$-algebras, this allows one to compute the $K_{0}$-groups and $K_{1}$-groups in the above exact sequence. Moreover, since the $C^{*}$-algebra of a graph satisfying Condition (K) has real rank zero [9, Theorem 3.5], it follows from [3] that the descending connecting map $\partial_{0}: K_{0}\left(C^{*}(E) / J\right) \rightarrow K_{1}(J)$ is the zero map. All that remains is to describe a method for computing the other connecting group homomorphisms.

The purpose of this paper is to provide explicit formulae for computing the six-term exact sequence, the main challenge being to compute the connecting map $\partial_{1}: K_{1}\left(C^{*}(E) / J\right) \rightarrow K_{0}(J)$. We shall also show that $\partial_{0}: K_{0}\left(C^{*}(E) / J\right) \rightarrow K_{1}(J)$ is the zero map regardless of whether the graph $E$ satisfies Condition $(\mathrm{K})$ or not. All our calculations hold for an arbitrary graph algebra $C^{*}(E)$ and an arbitrary gauge-invariant ideal $J$ in $C^{*}(E)$, even in the case of so-called breaking vertices.

To compute $\partial_{1}$, we need to choose generators for the $K$-groups involved. There is a canonical (and well-known) way to do this in $K_{0}$; one can choose an isomorphism of $K_{0}\left(C^{*}(E)\right)$ with $\operatorname{coker}\left(A^{t}-I\right)$ taking $\left[p_{v}\right]$ to $\mathbf{e}_{v}+\operatorname{Im}\left(A^{t}-\right.$ $I)$, where $\mathbf{e}_{v}$ is the vector with a 1 in the $v^{\text {th }}$ position and zeroes elsewhere. However, for the $K_{1}$-group the calculation is substantially harder. Descriptions of $K_{1}$ can be found in [2] and [5], but we need a more explicit description and therefore choose a different approach, choosing explicit generators for $K_{1}$ based on a slightly intricate indexing of the entries in a matrix over $C^{*}(E)$. Although any quotient of a graph $C^{*}$-algebra by a gauge-invariant ideal is isomorphic to a graph $C^{*}$-algebra, it will be more convenient for us to use that such a quotient is isomorphic to a relative graph $C^{*}$-algebra (cf. [11]), and we will therefore find generators of $K_{0}$ and $K_{1}$, not just for graph $C^{*}$-algebras, but for relative graph $C^{*}$-algebras.

We prove that the generators we choose for $K_{1}$ are indeed generators by computing the index map of the canonical Toeplitz extension of $C^{*}(E)$, using methods developed by Katsura in that framework. Our approach involves computing the index map using the canonical method (cf. [14]) of lifting the generating unitaries to partial isometries and computing defects. This method has similarities with the approach for Cuntz-Krieger algebras outlined by Cuntz himself in [4], and discussed with a few more details 
in [13. After describing how to choose generators for $K_{0}$ and $K_{1}$ of any relative graph $C^{*}$-algebra, we determine the index map $\partial_{1}: K_{1}\left(C^{*}(E) / J\right) \rightarrow$ $K_{0}(J)$ by, in a new extension, again lifting our generating unitaries to partial isometries, and computing defects.

In Section 2 we briefly introduce graph $C^{*}$-algebras, relative graph $C^{*}$ algebras, and gauge-invariant ideals of graph $C^{*}$-algebras. In Section 3 we find generators of $K_{0}$ and $K_{1}$ of any relative graph $C^{*}$-algebra. Section 4 states the main result of the paper, allowing the computation of the index map $\partial_{1}: K_{1}\left(C^{*}(E) / J\right) \rightarrow K_{0}(J)$ and the other maps in the six-term exact sequence (1.1), and this result is proved in Section 5 .

\section{Preliminaries}

A (directed) graph $E=\left(E^{0}, E^{1}, r, s\right)$ consists of a countable set $E^{0}$ of vertices, a countable set $E^{1}$ of edges, and maps $r, s: E^{1} \rightarrow E^{0}$ identifying the range and source of each edge. A vertex $v \in E^{0}$ is called a sink if $\left|s^{-1}(v)\right|=0$, and $v$ is called an infinite emitter if $\left|s^{-1}(v)\right|=\infty$. A graph $E$ is said to be row-finite if it has no infinite emitters. If $v$ is either a sink or an infinite emitter, then we call $v$ a singular vertex. We write $E_{\text {sing }}^{0}$ for the set of singular vertices. Vertices that are not singular vertices are called regular vertices and we write $E_{\mathrm{reg}}^{0}$ for the set of regular vertices.

If $E$ is a graph, a Cuntz-Krieger E-family is a set of mutually orthogonal projections $\left\{p_{v}: v \in E^{0}\right\}$ and a set of partial isometries $\left\{s_{e}: e \in E^{1}\right\}$ with mutually orthogonal ranges which satisfy the Cuntz-Krieger relations:

(CK1) $s_{e}^{*} s_{e}=p_{r(e)}$ for every $e \in E^{1}$;

(CK2) $p_{v}=\sum_{s(e)=v} s_{e} s_{e}^{*}$ for every $v \in E_{\mathrm{reg}}^{0}$;

(CK3) $s_{e} s_{e}^{*} \leq p_{s(e)}$ for every $e \in E^{1}$.

The graph algebra $C^{*}(E)$ is defined to be the $C^{*}$-algebra generated by a universal Cuntz-Krieger $E$-family.

It will in this paper also be relevant to work with relative graph $C^{*}$ algebras introduced in [11]. To define a relative graph $C^{*}$-algebra we must, in addition to a graph $E$, specify a subset $R$ of $E_{\mathrm{reg}}^{0}$. A Cuntz-Krieger $(E, R)$-family is then a set of mutually orthogonal projections $\left\{p_{v}: v \in E^{0}\right\}$ and a set of partial isometries $\left\{s_{e}: e \in E^{1}\right\}$ with mutually orthogonal ranges which satisfy the relations (CK1) and (CK3) above together with the following relative Cuntz-Krieger relation:

$(\mathrm{RCK} 2) p_{v}=\sum_{s(e)=v} s_{e} s_{e}^{*}$ for every $v \in R$.

The relative graph algebra $C^{*}(E, R)$ is defined to be the $C^{*}$-algebra generated by a universal Cuntz-Krieger $(E, R)$-family. If $R=E_{\mathrm{reg}}^{0}$, then a Cuntz-Krieger $(E, R)$-family is the same as a Cuntz-Krieger $E$-family and $C^{*}(E, R)=C^{*}(E)$. If $R=\emptyset$, then $C^{*}(E, R)$ is the Toeplitz algebra $\mathcal{T}(E)$ defined in [8, Theorem 4.1]. We will call a Cuntz-Krieger $(E, \emptyset)$-family a Toeplitz-Cuntz-Krieger E-family. 
A path in $E$ is a sequence of edges $\alpha=\alpha_{1} \alpha_{2} \ldots \alpha_{n}$ with $r\left(\alpha_{i}\right)=s\left(\alpha_{i+1}\right)$ for $1 \leq i<n$, and we say that $\alpha$ has length $|\alpha|=n$. We let $E^{n}$ denote the set of all paths of length $n$, and we let $E^{*}:=\bigcup_{n=0}^{\infty} E^{n}$ denote the set of finite paths in $E$. Note that vertices are considered paths of length zero. The maps $r, s$ extend to $E^{*}$, and for $v, w \in E^{0}$ we write $v \geq w$ if there exists a path $\alpha \in E^{*}$ with $s(\alpha)=v$ and $r(\alpha)=w$. Also for a path $\alpha:=\alpha_{1} \ldots \alpha_{n}$ we define $s_{\alpha}:=s_{\alpha_{1}} \ldots s_{\alpha_{n}}$, and for a vertex $v \in E^{0}$ we let $s_{v}:=p_{v}$. It is a consequence of the relations (CK1) and (CK3) that $C^{*}(E, R)=\overline{\operatorname{span}}\left\{s_{\alpha} s_{\beta}^{*}: \alpha, \beta \in E^{*}\right.$ and $\left.r(\alpha)=r(\beta)\right\}$.

We say that a path $\alpha:=\alpha_{1} \ldots \alpha_{n}$ of length 1 or greater is a cycle if $r(\alpha)=s(\alpha)$, and we call the vertex $s(\alpha)=r(\alpha)$ the base point of the cycle. A cycle is said to be simple if $s\left(\alpha_{i}\right) \neq s\left(\alpha_{1}\right)$ for all $1<i \leq n$. The following is an important condition in the theory of graph $C^{*}$-algebras.

Condition (K): No vertex in $E$ is the base point of exactly one simple cycle; that is, every vertex is either the base point of no cycles or at least two simple cycles.

For any graph $E$ a subset $H \subseteq E^{0}$ is hereditary if whenever $v, w \in E^{0}$ with $v \in H$ and $v \geq w$, then $w \in H$. A hereditary subset $H$ is saturated if whenever $v \in E_{\text {reg }}^{0}$ with $r\left(s^{-1}(v)\right) \subseteq H$, then $v \in H$. For any saturated hereditary subset $H$, the breaking vertices corresponding to $H$ are the elements of the set

$$
B_{H}:=\left\{v \in E^{0}:\left|s^{-1}(v)\right|=\infty \text { and } 0<\left|s^{-1}(v) \cap r^{-1}\left(E^{0} \backslash H\right)\right|<\infty\right\} .
$$

An admissible pair $(H, S)$ consists of a saturated hereditary subset $H$ and a subset $S \subseteq B_{H}$. For a fixed graph $E$ we order the collection of admissible pairs for $E$ by defining $(H, S) \leq\left(H^{\prime}, S^{\prime}\right)$ if and only if $H \subseteq H^{\prime}$ and $S \subseteq$ $H^{\prime} \cup S^{\prime}$. For any admissible pair $(H, S)$ we define $J_{(H, S)}$ to be the ideal in $C^{*}(E)$ generated by

$$
\left\{p_{v}: v \in H\right\} \cup\left\{p_{v_{0}}^{H}: v_{0} \in S\right\},
$$

where $p_{v_{0}}^{H}$ is the gap projection defined by

$$
p_{v_{0}}^{H}:=p_{v_{0}}-\sum_{\begin{array}{c}
s(e)=v_{0} \\
r(e) \notin H
\end{array}} s_{e} s_{e}^{*} .
$$

Note that the definition of $B_{H}$ ensures that the sum on the right is finite.

For any graph $E$ there is a canonical gauge action $\gamma: \mathbb{T} \rightarrow \operatorname{Aut} C^{*}(E)$ with the property that for any $z \in \mathbb{T}$ we have $\gamma_{z}\left(p_{v}\right)=p_{v}$ for all $v \in E^{0}$ and $\gamma_{z}\left(s_{e}\right)=z s_{e}$ for all $e \in E^{1}$. We say that an ideal $J \triangleleft C^{*}(E)$ is gauge invariant if $\gamma_{z}(J) \subseteq J$ for all $z \in \mathbb{T}$.

There is a bijective correspondence between the lattice of admissible pairs of $E$ and the lattice of gauge-invariant ideals of $C^{*}(E)$ given by $(H, S) \mapsto$ $J_{(H, S)}\left[2\right.$, Theorem 3.6]. When $E$ satisfies Condition $(\mathrm{K})$, all ideals of $C^{*}(E)$ 
are gauge invariant [2, Corollary 3.8] and the map $(H, S) \mapsto J_{(H, S)}$ is onto the lattice of ideals of $C^{*}(E)$. When $B_{H}=\emptyset$, we write $J_{H}$ in place of $J_{(H, \emptyset)}$ and observe that $J_{H}$ equals the ideal generated by $\left\{p_{v}: v \in H\right\}$. Note that if $E$ is row-finite, then $B_{H}$ is empty for every saturated hereditary subset $H$.

\section{3. $K$-THEORY FOR RELATIVE GRAPH ALGEBRAS}

For a graph $E$, the adjacency matrix is the $E^{0} \times E^{0}$ matrix $A_{E}$ with

$$
A_{E}(v, w):=\#\left\{e \in E^{1}: s(e)=v \text { and } r(e)=w\right\} .
$$

Note that the entries of $A_{E}$ are elements of $\{0,1,2, \ldots\} \cup\{\infty\}$. Writing the adjacency matrix with respect to the decomposition $E^{0}=E_{\text {reg }}^{0} \sqcup E_{\text {sing }}^{0}$, where the regular vertices are listed first, we obtain a (possibly infinite) block matrix

$$
A_{E}=\left[\begin{array}{cc}
A & \alpha \\
H & \eta
\end{array}\right]
$$

in which all entries of $A$ and $\alpha$ are finite, but the entries in $H$ and $\eta$ may be infinite. We will often just substitute "*" for $H$ and $\eta$, as they turn out to be irrelevant for the $K$-theory. Indeed, by [2] and [5] we know that the map

$$
\left[\begin{array}{c}
A^{t}-I \\
\alpha^{t}
\end{array}\right]: \mathbb{Z}^{E_{\text {reg }}^{0}} \rightarrow \mathbb{Z}^{E^{0}}
$$

contains the needed information, as

$$
K_{0}\left(C^{*}(E)\right) \simeq \operatorname{coker}\left[\begin{array}{c}
A^{t}-I \\
\alpha^{t}
\end{array}\right] \quad K_{1}\left(C^{*}(E)\right) \simeq \operatorname{ker}\left[\begin{array}{c}
A^{t}-I \\
\alpha^{t}
\end{array}\right] .
$$

This result can be generalized to relative graph $C^{*}$-algebras. In fact, we prove in Proposition 3.8 that if $E$ is a graph, $R \subseteq E_{\mathrm{reg}}$, and $A_{E}=\left[\begin{array}{cc}A & \alpha \\ H & \eta\end{array}\right]$ is the adjacency matrix of $E$ written with respect to the decomposition $E^{0}=R \sqcup\left(E^{0} \backslash R\right)$, where the vertices belonging to $R$ are listed first, then there exists a group isomorphism $\chi_{0}:$ coker $\left[\begin{array}{c}A^{t}-I \\ \alpha^{t}\end{array}\right] \rightarrow K_{0}\left(C^{*}(E, R)\right)$ given for any $v \in R$ by

$$
\chi_{0}\left(\mathbf{e}_{v}+\operatorname{im}\left[\begin{array}{c}
A^{t}-I \\
\alpha^{t}
\end{array}\right]\right)=\left[p_{v}\right]_{0},
$$

and we construct a similar group isomorphism $\chi_{1}$ between $\operatorname{ker}\left[\begin{array}{c}A^{t}-I \\ \alpha^{t}\end{array}\right]$ and $K_{1}\left(C^{*}(E, R)\right)$. For this we first introduce some notation:

Given $\mathbf{x} \in \operatorname{ker}\left[\begin{array}{c}A^{t}-I \\ \alpha^{t}\end{array}\right]$, first note that by definition $\mathbf{x}$ has only finitely many nonzero entries $x_{v_{1}}, \ldots x_{v_{k}}$. We define

$$
\begin{aligned}
& L_{\mathbf{x}}^{+}:=\left\{(e, i): e \in E^{1}, 1 \leq i \leq-x_{s(e)}\right\} \cup\left\{(v, i): v \in E^{0}, 1 \leq i \leq x_{v}\right\} \\
& L_{\mathbf{x}}^{-}:=\left\{(e, i): e \in E^{1}, 1 \leq i \leq x_{s(e)}\right\} \cup\left\{(v, i): v \in E^{0}, 1 \leq i \leq-x_{v}\right\}
\end{aligned}
$$

and note, using the convention that $r(v)=v$ for any $v \in E^{0}$, that 
Lemma 3.1. When $\mathbf{x} \in \operatorname{ker}\left[\begin{array}{c}A^{t}-I \\ \alpha^{t}\end{array}\right]$, then for any vertex $v \in E^{0}$ the sets

$$
L_{v}^{+}=\left\{(x, i) \in L_{\mathbf{x}}^{+}: r(x)=v\right\}
$$

and

$$
L_{v}^{-}=\left\{(x, i) \in L_{\mathbf{x}}^{-}: r(x)=v\right\}
$$

are finite and have the same number of elements.

Proof. We need to consider three cases separately.

CASE I: $v \in R$ and $x_{v} \geq 0$.

The number of elements in $L_{v}^{+}$is

$$
x_{v}+\sum_{x_{w}<0} \#\left\{e \in E^{1}: s(e)=w, r(e)=v\right\} \cdot\left(-x_{w}\right)=x_{v}-\sum_{x_{w}<0} A_{v, w}^{t} x_{w}
$$

and the number of elements in $L_{v}^{-}$is

$$
\sum_{x_{w}>0} \#\left\{e \in E^{1}: s(e)=w, r(e)=v\right\} \cdot x_{w}=\sum_{x_{w}>0} A_{v, w}^{t} x_{w}
$$

so the claim follows by inspecting the $v$ coordinate of the equality $A^{t} \mathbf{x}=\mathbf{x}$. CASE II: $v \in R$ and $x_{v}<0$.

As above.

CASE III: $v \in E^{0} \backslash R$.

The number of elements in $L_{v}^{+}$is

$$
\sum_{x_{w}<0} \#\left\{e \in E^{1}: s(e)=w, r(e)=v\right\} \cdot\left(-x_{w}\right)=-\sum_{x_{w}<0} \alpha_{v, w}^{t} x_{w}
$$

and the number of elements in $L_{v}^{-}$is

$$
\sum_{x_{w}>0} \#\left\{e \in E^{1}: s(e)=w, r(e)=v\right\} \cdot x_{w}=\sum_{x_{w}>0} \alpha_{v, w}^{t} x_{w}
$$

so the claim follows by inspecting the $v$ coordinate of the equality $\alpha^{t} \mathbf{x}=$ 0 .

Lemma 3.2. $L_{\mathbf{x}}^{+}$and $L_{\mathbf{x}}^{-}$are finite sets, and have the same number of elements.

Proof. This follows from Lemma 3.1, as indeed $L_{v}^{+} \neq \emptyset$ only when $v$ lies in the set

$$
\left\{v: x_{v} \neq 0\right\} \cup\left\{v: x_{w} \neq 0 \text { for some } w \in s\left(r^{-1}(v)\right)\right\}
$$

which is finite since no $w$ is an infinite emitter.

Denote the common number of elements in $L_{\mathbf{x}}^{+}$and $L_{\mathbf{x}}^{-}$by $h$. Because of Lemma 3.1, we can define bijections

$$
[\cdot]: L_{\mathbf{x}}^{+} \rightarrow\{1, \ldots, h\} \quad\langle\cdot\rangle: L_{\mathbf{x}}^{-} \rightarrow\{1, \ldots, h\}
$$

with the property that

$$
[x, i]=\langle y, j\rangle \Longrightarrow r(x)=r(y)
$$


with the convention $r(v)=v$.

When $\mathfrak{A}$ is a $C^{*}$-algebra then we let $\mathrm{M}_{h}(\mathfrak{A})$ denote the $C^{*}$-algebra of $h \times h$-matrices over $\mathfrak{A}$. We are ready for our key definitions:

Definition 3.3. Suppose that $\mathfrak{A}$ is a $C^{*}$-algebra which contains a ToeplitzCuntz-Krieger $E$-family $\left\{p_{v}: v \in E^{0}\right\} \cup\left\{s_{e}: e \in E^{1}\right\}$. With notation as above, we define the two elements $V, P \in \mathrm{M}_{h}(\mathfrak{A})$ by

$$
V=\sum_{\substack{1 \leq i \leq x_{w} \\ s(e)=w}} s_{e} \mathrm{E}_{[w, i],\langle e, i\rangle}+\sum_{\substack{1 \leq i \leq-x_{w} \\ s(e)=w}} s_{e}^{*} \mathrm{E}_{[e, i],\langle w, i\rangle}
$$

and

$$
P=\sum_{1 \leq i \leq x_{w}} p_{w} \mathrm{E}_{[w, i],[w, i]}+\sum_{\substack{1 \leq i \leq-x_{w} \\ s(e)=w, r(e)=v}} p_{v} \mathrm{E}_{[e, i],[e, i]}
$$

Here $\mathrm{E}_{\bullet, \bullet}$ denote the standard matrix units in $\mathrm{M}_{h}(M(\mathfrak{A}))$ where $M(\mathfrak{A})$ is the multiplier algebra of $\mathfrak{A}$.

Lemma 3.4. If $\left\{s_{e}, p_{v}: e \in E^{1}, v \in E^{0}\right\}$ is a Toeplitz-Cuntz-Krieger Efamily, then

$$
\begin{aligned}
P & =\sum_{\substack{1 \leq i \leq-x_{w} \\
V^{*}}} p_{w} \mathrm{E}_{\langle w, i\rangle,\langle w, i\rangle}+\sum_{\substack{1 \leq i \leq x_{w} \\
s(e)=w, r(e)=v}} p_{v} \mathrm{E}_{\langle e, i\rangle,\langle e, i\rangle}, \\
V^{*} & \sum_{\substack{1 \leq i \leq x_{w} \\
s(e)=w}} s_{e}^{*} \mathrm{E}_{\langle e, i\rangle,[w, i]}+\sum_{\substack{1 \leq i \leq-x_{w} \\
s(e)=w}} \mathrm{E}_{\langle w, i\rangle,[e, i]}, \\
V V^{*}= & \sum_{\substack{1 \leq i \leq x_{w} \\
s(e)=w}} s_{e} s_{e}^{*} \mathrm{E}_{[w, i],[w, i]}+\sum_{\substack{1 \leq i \leq-x_{w} \\
s(e)=w, r(e)=v}} p_{v} \mathrm{E}_{[e, i],[e, i]}, \\
V^{*} V & \sum_{\substack{1 \leq i \leq-x_{w} \\
s(e)=w}} s_{e} s_{e}^{*} \mathrm{E}_{\langle w, i\rangle,\langle w, i\rangle}+\sum_{\substack{1 \leq i \leq x_{w} \\
s(e)=w, r(e)=v}} p_{v} \mathrm{E}_{\langle e, i\rangle,\langle e, i\rangle} .
\end{aligned}
$$

Proof. It follows from Lemma 3.2 and Equation (3.1) that

$$
\sum_{(x, i) \in L_{\mathbf{x}}^{+}} p_{r(x)} \mathrm{E}_{[x, i],[x, i]}=\sum_{(x, i) \in L_{\mathbf{x}}^{-}} p_{r(x)} \mathrm{E}_{\langle x, i\rangle,\langle x, i\rangle},
$$

and it is easy to check that

$$
P=\sum_{(x, i) \in L_{\mathbf{x}}^{+}} p_{r(x)} \mathrm{E}_{[x, i],[x, i]}
$$

and that

$$
\sum_{(x, i) \in L_{\mathbf{x}}^{-}} p_{r(x)} \mathrm{E}_{\langle x, i\rangle,\langle x, i\rangle}=\sum_{1 \leq i \leq-x_{w}} p_{w} \mathrm{E}_{\langle w, i\rangle,\langle w, i\rangle}+\sum_{\substack{1 \leq i \leq x_{w} \\ s(e)=w, r(e)=v}} p_{v} \mathrm{E}_{\langle e, i\rangle,\langle e, i\rangle}
$$


from which Equation (3.2) then follows. Equation (3.3) is straightforward to check. For Equation (3.4), using only (3.3) and the matrix unit relations we get that

$$
V V^{*}=\sum_{\substack{1 \leq i \leq x_{w} \\
s(e)=w}} s_{e} s_{e}^{*} \mathrm{E}_{[w, i],[w, i]}+\sum_{\begin{array}{c}
1 \leq i \leq-x_{w} \\
s(e)=w, r(e)=v \\
s\left(e^{\prime}\right)=w, r\left(e^{\prime}\right)=v^{\prime}
\end{array}} s_{e}^{*} s_{e^{\prime}} \mathrm{E}_{[e, i],\left[e^{\prime}, i\right]}
$$

and (3.4) holds from (CK1) and the fact that the $s_{e}$ 's have mutually orthogonal ranges. The computation for $V^{*} V$ is similar.

Lemma 3.5. If $\left\{s_{e}, p_{v}: e \in E^{1}, v \in E^{0}\right\}$ is a Toeplitz-Cuntz-Krieger Efamily, then $V$ is a partial isometry with $P V=V P=V$.

Proof. Using Equation (3.5), the definition of $V$, and the fact that the $s_{e}$ 's are partial isometries, we see that $V V^{*} V=V$, so that $V$ is a partial isometry. Furthermore, (CK3) implies $P V=V$ and $V P=V$ by Equation (3.2).

We now let $\left\{s_{e}, p_{v}: e \in E^{1}, v \in E^{0}\right\}$ be the universal Cuntz-Krieger $(E, R)$-family generating $C^{*}(E, R)$ and write $\mathrm{V}_{\mathbf{x}}$ and $\mathrm{P}_{\mathbf{x}}$ for the corresponding elements $V$ and $P$ in $\mathrm{M}_{h}\left(C^{*}(E, R)\right)$ defined in Definition 3.3. using the added subscript to emphasize the dependence of each of $V$ and $P$ on $\mathbf{x} \in \operatorname{ker}\left[\begin{array}{c}A^{t}-1 \\ \alpha^{t}\end{array}\right]$. In addition, we define $\mathrm{U}_{\mathbf{x}}:=\mathrm{V}_{\mathbf{x}}+\left(1-\mathrm{P}_{\mathbf{x}}\right)$.

Fact 3.6. We have that $\mathrm{V}_{\mathbf{x}} \mathrm{V}_{\mathbf{x}}^{*}=\mathrm{V}_{\mathbf{x}}^{*} \mathrm{~V}_{\mathbf{x}}=\mathrm{P}_{\mathbf{x}}$, and hence that $\mathrm{U}_{\mathbf{x}}$ is a unitary. Proof. It follows from Equation (3.4) and (RCK2) that

$$
\begin{aligned}
\mathrm{V}_{\mathbf{x}} \mathrm{V}_{\mathbf{x}}^{*} & =\sum_{1 \leq i \leq x_{w}}\left(\sum_{s(e)=w} s_{e} s_{e}^{*}\right) \mathrm{E}_{[w, i],[w, i]}+\sum_{\substack{1 \leq i \leq-x_{w} \\
s(e)=w, r(e)=v}} p_{v} \mathrm{E}_{[e, i],[e, i]} \\
& =\sum_{1 \leq i \leq x_{w}} p_{w} \mathrm{E}_{[w, i],[w, i]}+\sum_{\substack{1 \leq i \leq-x_{w} \\
s(e)=w, r(e)=v}} p_{v} \mathrm{E}_{[e, i],[e, i]} \\
& =\mathbf{P}_{\mathbf{x}}
\end{aligned}
$$

showing the first claim. Likewise, Equation (3.5) and (RCK2) show that $\mathrm{V}_{\mathbf{x}}^{*} \mathrm{~V}_{\mathbf{x}}=\mathrm{P}_{\mathbf{x}}$. The fact that $\mathrm{U}_{\mathbf{x}}$ is a unitary follows.

Remark 3.7. Notice that although $\mathrm{U}_{\mathbf{x}}$ does depend on the choice of bijections

$$
[\cdot]: L_{\mathbf{x}}^{+} \rightarrow\{1, \ldots, h\} \quad\langle\cdot\rangle: L_{\mathbf{x}}^{-} \rightarrow\{1, \ldots, h\},
$$

the element $\left[\mathrm{U}_{\mathbf{x}}\right]_{1}$ of $K_{1}\left(C^{*}(E, R)\right)$ does not.

Proposition 3.8. Let $E$ be a graph, let $V$ be a subset of $E_{\mathrm{reg}}$ and let

$$
A_{E}=\left[\begin{array}{cc}
A & \alpha \\
H & \eta
\end{array}\right]
$$

be the adjacency matrix of $E$ written with respect to the decomposition $E^{0}=$ $V \sqcup\left(E^{0} \backslash V\right)$ where the vertices belonging to $V$ are listed first. 
(1) There exists a group isomorphism $\chi_{0}$ : coker $\left[\begin{array}{c}A^{t}-I \\ \alpha^{t}\end{array}\right] \rightarrow K_{0}\left(C^{*}(E, R)\right)$ given for any $v \in E^{0}$ by

$$
\chi_{0}\left(\mathbf{e}_{v}+\operatorname{im}\left[\begin{array}{c}
A^{t}-I \\
\alpha^{t}
\end{array}\right]\right)=\left[p_{v}\right]_{0} .
$$

The preimage of the positive cone of $K_{0}\left(C^{*}(E, R)\right)$ is generated by $\left\{\mathbf{e}_{v}: v \in E^{0}\right\} \cup\left\{\mathbf{e}_{v}-\sum_{e \in F} \mathbf{e}_{r(e)}: v \in E_{\text {sing }}^{0}, F \subseteq s^{-1}(v), F\right.$ finite $\}$.

(2) The map $\chi_{1}$ : $\operatorname{ker}\left[\begin{array}{c}A^{t}-I \\ \alpha^{t}\end{array}\right] \rightarrow K_{1}\left(C^{*}(E, R)\right)$ given by

$$
\chi_{1}(\mathbf{x})=\left[\mathrm{U}_{\mathbf{x}}\right]_{1}
$$

is group isomorphism.

Proof. As noted in [11, we can realize $C^{*}(E, R)$ as a relative Cuntz-Pimsner algebra over a Hilbert bimodule $\mathcal{X}_{E}$. It is not difficult to check that the corresponding Toeplitz algebra $\mathcal{T}_{\mathcal{X}_{E}}$ is isomorphic to the Toeplitz algebra $\mathcal{T}(E)$. We let $\pi: \mathcal{T}(E) \rightarrow C^{*}(E, R)$ denote the canonical map, so that

$$
0 \longrightarrow \operatorname{ker} \pi \stackrel{\iota}{\longrightarrow} \mathcal{T}(E) \stackrel{\pi}{\longrightarrow} C^{*}(E, R) \longrightarrow 0
$$

is exact. The associated $K$-theory is then

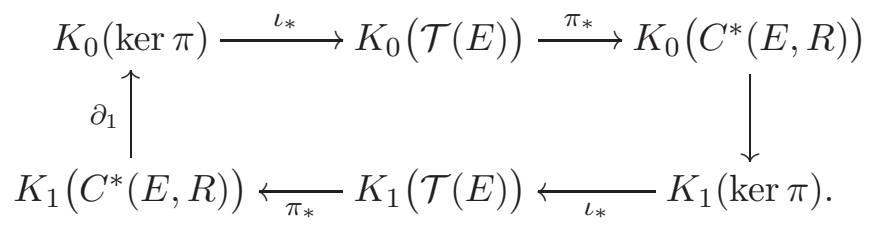

Now we appeal to Katsura's work. It follows from the results of [10, §8], that $\operatorname{ker} \pi$ and $\mathcal{T}(E)$ are $K K$-equivalent to the commutative $A F$-algebras $c_{0}(R)$ and $c_{0}\left(E^{0}\right)$, respectively, and that there are group isomorphisms $\kappa$ : $K_{0}(\operatorname{ker} \pi) \rightarrow \mathbb{Z}^{R}$ and $\lambda: K_{0}(\mathcal{T}(E)) \rightarrow \mathbb{Z}^{E^{0}}$ such that the diagram

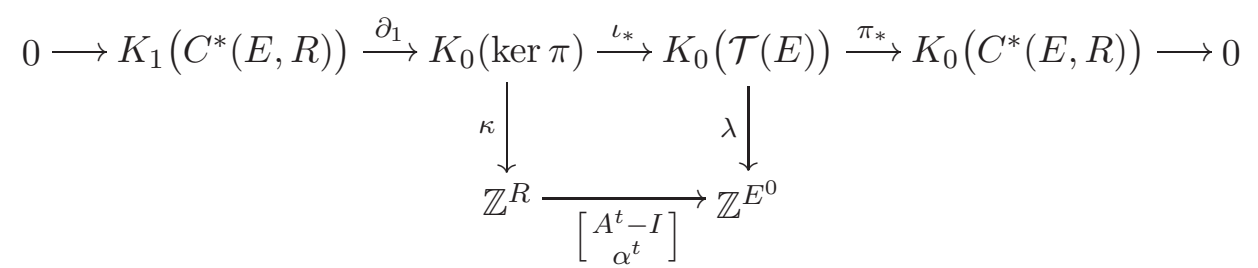

commutes with the top row exact. In [10] concrete $*$-homomorphisms are given inducing $\kappa$ and $\lambda$, but we do not need them here. All we need is the fact that $\lambda\left(p_{v}\right)=\mathbf{e}_{v}$ and

$$
\kappa\left(\left[p_{w}-\sum_{s(e)=w} s_{e} s_{e}^{*}\right]_{0}\right)=\mathbf{e}_{w}
$$


for $v \in E^{0}$ and $w \in R$. It follows that $\pi_{*} \circ \lambda^{-1}$ is a surjective group homomorphism from $\mathbb{Z}^{E^{0}}$ to $K_{0}\left(C^{*}(E, R)\right)$ which for any $v \in E^{0}$ maps $\mathbf{e}_{v}$ to $\left[p_{v}\right]_{0}$ and whose kernel is $\operatorname{im}\left[\begin{array}{c}A^{t}-I \\ \alpha^{t}\end{array}\right]$. The existence of a group isomorphism $\chi_{0}$ : coker $\left[\begin{array}{c}A^{t}-I \\ \alpha^{t}\end{array}\right] \rightarrow K_{0}\left(C^{*}(E, R)\right)$ which for any $v \in E^{0}$ satisfies Equation (3.6) follows from this. The description of the positive cone in the row-finite case was given in [1, Theorem 7.1]. For the general situation, it is shown in [15. Theorem 2.2] that the process of desingularization can be used to extend the result from the row-finite case to the general case.

To see that $\chi_{1}: \operatorname{ker}\left[\begin{array}{c}A^{t}-I \\ \alpha^{t}\end{array}\right] \rightarrow K_{1}\left(C^{*}(E, R)\right)$ is a group isomorphism, fix $\mathbf{x} \in \operatorname{ker}\left[\begin{array}{c}A^{t}-I \\ \alpha^{t}\end{array}\right]$ and lift $\mathrm{U}_{\mathbf{x}}=\mathrm{V}_{\mathbf{x}}+\left(1-\mathrm{P}_{\mathbf{x}}\right) \in \mathrm{M}_{h}\left(C^{*}(E, R)\right)$ to $\widetilde{\mathrm{U}}_{\mathbf{x}}=$ $\widetilde{\mathrm{V}}_{\mathbf{x}}+\left(1-\widetilde{\mathrm{P}}_{\mathbf{x}}\right) \in \mathrm{M}_{h}(\mathcal{T}(E))$ where $\widetilde{\mathrm{V}}_{\mathbf{x}}$ and $\widetilde{\mathrm{P}}_{\mathbf{x}}$ are the elements $V$ and $P$ in $\mathrm{M}_{h}(\mathcal{T}(E))$ we get by using the universal Toeplitz-Cuntz-Krieger $E$-family which generates $\mathcal{T}(E)$ in Definition 3.3, By Lemma 3.5, $\widetilde{\mathrm{V}}_{\mathbf{x}}$ is a partial isometry with $\widetilde{P}_{\mathbf{x}} \widetilde{V}_{\mathbf{x}}=\widetilde{V}_{\mathbf{x}} \widetilde{P}_{\mathbf{x}}=\widetilde{\mathrm{V}}_{\mathbf{x}}$. It follows that $\widetilde{\mathrm{U}}_{\mathbf{x}}$ is also a partial isometry. We need to compute the defect of $\widetilde{U}_{\mathbf{x}}$ as an element of $K_{0}(\operatorname{ker} \pi)$. We have by Lemma 3.4 that

$$
1-\widetilde{\mathrm{U}}_{\mathbf{x}} \widetilde{\mathrm{U}}_{\mathbf{x}}^{*}=\widetilde{\mathrm{P}}_{\mathbf{x}}-\widetilde{\mathrm{V}}_{\mathbf{x}} \widetilde{\mathrm{V}}_{\mathbf{x}}^{*}=\sum_{1 \leq i \leq x_{w}}\left(p_{w}-\sum_{s(e)=w} s_{e} s_{e}^{*}\right) \mathrm{E}_{[w, i],[w, i]}
$$

and a similar equation for $1-\widetilde{\mathrm{U}}_{\mathbf{x}}^{*} \widetilde{\mathrm{U}}_{\mathbf{x}}$. Hence, in $K_{0}(\operatorname{ker} \pi)$ we have that

$$
\left[1-\widetilde{\mathbf{U}}_{\mathbf{x}} \widetilde{\mathrm{U}}_{\mathbf{x}}^{*}\right]_{0}-\left[1-\widetilde{\mathbf{U}}_{\mathbf{x}}^{*} \widetilde{U}_{\mathbf{x}}\right]_{0}=\sum_{x_{w} \neq 0} x_{w}\left[p_{w}-\sum_{s(e)=w} s_{e} s_{e}^{*}\right]_{0}
$$

which together with Equation (3.8) and Equation (3.9) implies that

$$
\kappa \circ \partial_{1} \circ \chi_{1}(\mathbf{x})=\mathbf{x}
$$

for any $\mathbf{x} \in\left[\begin{array}{c}A^{t}-I \\ \alpha^{t}\end{array}\right]$. This shows that $\chi_{1}$ is injective. Let us also prove that $\chi_{1}$ is a group isomorphism. Fix $\mathbf{y} \in K_{1}\left(C^{*}(E, R)\right)$ and note that

$$
\left[\begin{array}{c}
A^{t}-I \\
\alpha^{t}
\end{array}\right] \circ \kappa \circ \partial_{1}(\mathbf{y})=\lambda \circ \iota_{*} \circ \partial_{1}(\mathbf{y})=0
$$

so that $\mathbf{z}:=\kappa \circ \partial_{1}(\mathbf{y})$ lies in $\operatorname{ker}\left[\begin{array}{c}A^{t}-I \\ \alpha^{t}\end{array}\right]$. Since $\kappa \circ \partial_{1}$ is injective, it follows from Equation (3.10) that $\chi_{1}(\mathbf{z})=\mathbf{y}$. We conclude that $\kappa \circ \partial_{1}$ is actually an inverse to $\chi_{1}$, and hence $\chi_{1}$ is a group isomorphism.

\section{THE INDEX MAP}

Let $E$ be a graph and let $J$ be a gauge-invariant ideal in $C^{*}(E)$. It follows from [2] that $J$ is of the form $J_{(H, S)}$ for an admissible pair $(H, S)$. Writing 
the adjacency matrix of $E$ with respect to the decomposition

$$
E_{\text {reg }}^{0} \cap H, \quad E_{\text {sing }}^{0} \cap H, \quad E_{\text {reg }}^{0} \backslash H, \quad E_{\text {sing }}^{0} \backslash(H \cup S), \quad S
$$

we arrive at the matrix

$$
\left[\begin{array}{lllll}
A & \alpha & 0 & 0 & 0 \\
* & * & 0 & 0 & 0 \\
X & \xi & B & \beta & \eta \\
* & * & * & * & * \\
* & * & \Gamma & \gamma & Z
\end{array}\right]
$$

We are now ready to state our main result. Here and below, whenever $T: G_{1} \rightarrow G_{2}$ is a group homomorphism between abelian groups and $H_{1}$ and $H_{2}$ are subgroups of $G_{1}$ and $G_{2}$, respectively, such that $T\left(H_{1}\right) \subseteq H_{2}$, then we also use $T$ to denote the group homomorphism from $G_{1} / H_{1}$ to $G_{2} / H_{2}$ induced by $T$, and we denote by $I_{a_{1} \cdots a_{k}}$ the canonical inclusion of the indicated components of a direct sum into a larger direct sum, and by $P_{a_{1} \cdots a_{k}}$ the corresponding projection.

Theorem 4.1. Let $E$ be a graph and let $(H, S)$ be an admissible pair. The six term exact sequence in $K$-theory

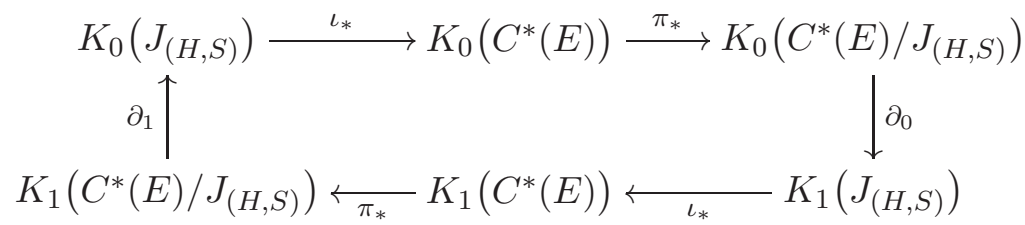

is isomorphic to

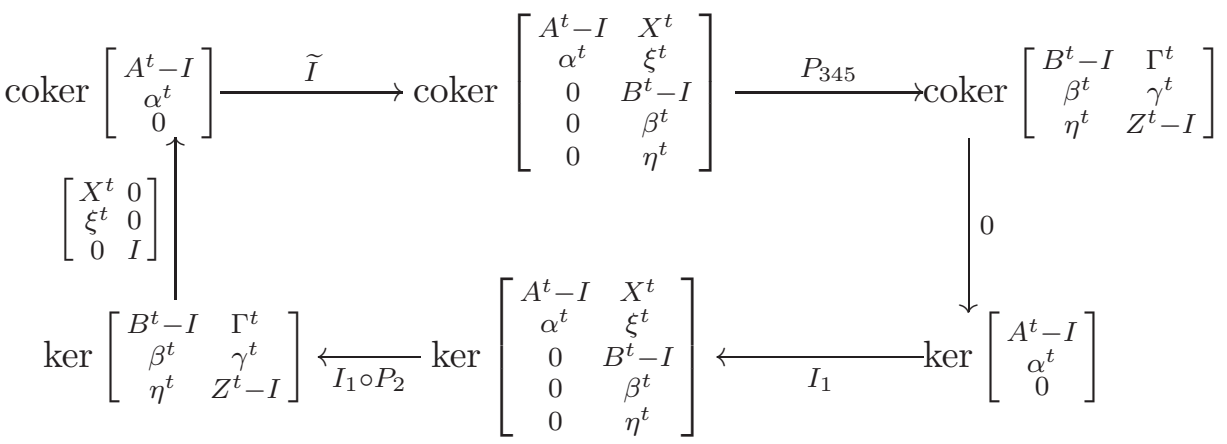

where $\widetilde{I}$ is given by the block matrix

$$
\left[\begin{array}{ccc}
I & 0 & 0 \\
0 & I & 0 \\
0 & 0 & -\Gamma^{t} \\
0 & 0 & -\gamma^{t} \\
0 & 0 & I-Z^{t}
\end{array}\right]=I_{125}-\left[\begin{array}{ccc}
0 & 0 & 0 \\
0 & 0 & 0 \\
0 & 0 & \Gamma^{t} \\
0 & 0 & \gamma^{t} \\
0 & 0 & Z^{t}
\end{array}\right]
$$


Each cokernel is ordered as described in Theorem 3.8. We postpone the proof of the theorem to the ensuing section, but remark here that the isomorphism between the two six term exact sequences is given by explicit defined maps which are described in the proof.

For now, let us record a number of examples and specializations:

Remark 4.2. If the saturated hereditary subset $H$ has no breaking vertices (this is always the case if $E$ is row-finite), or if $S=\emptyset$, then the six term exact sequence of Theorem 4.1 reduces to

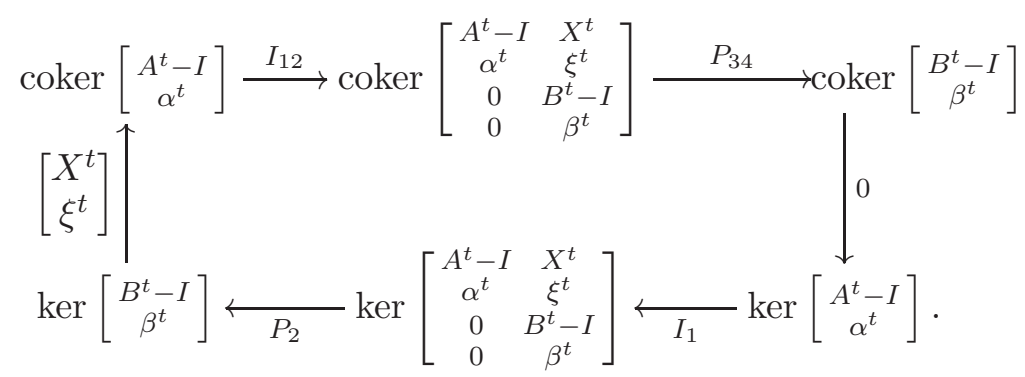

Remark 4.3. Let $E$ be a row-finite graph with no sinks. Then any gaugeinvariant ideal in $C^{*}(E)$ has the form $J_{H}$ for some saturated hereditary subset $H$ and the six term exact sequence of Theorem 4.1 reduces in this case to

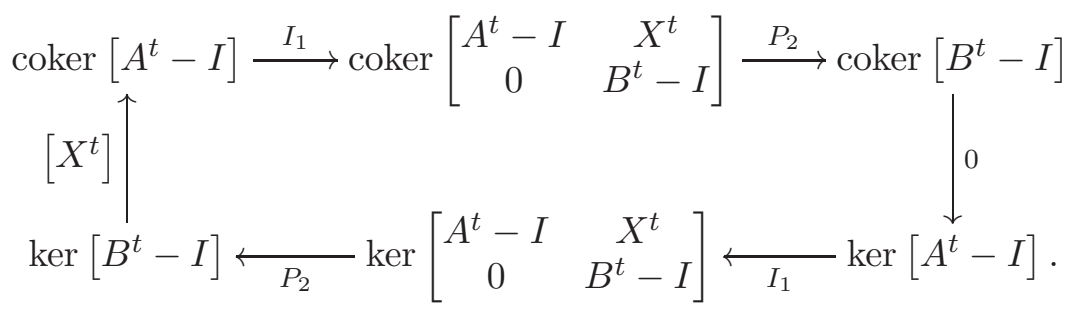

Corollary 4.4. Let $E$ be a graph such that the associated graph $C^{*}$-algebra $C^{*}(E)$ contains a unique proper nontrivial ideal. Then this ideal has the form $J_{H}$ for some saturated hereditary subset $H$ with no breaking vertices. Consequently, the cyclic six term exact sequence determined by the short exact sequence $0 \rightarrow J_{H} \rightarrow C^{*}(E) \rightarrow C^{*}(E) / J_{H} \rightarrow 0$ is isomorphic to the cyclic exact sequence described in (4.1).

Proof. If $E$ has a unique proper nontrivial ideal, then it follows from [7, Lemma 3.1] that the ideal has the form $J_{H}$ for a saturated hereditary subset $H$ with no breaking vertices.

Example 4.5. Consider the class of graphs $E_{x, y, z}$ given by the adjacency matrix

$$
\left[\begin{array}{llll}
0 & 0 & 0 & 0 \\
x & 1 & 1 & 0 \\
y & 1 & 1 & 1 \\
z & 0 & 1 & 1
\end{array}\right]
$$


where $x, y, z \in \mathbb{N}$. These graphs all satisfy Condition $(\mathrm{K})$ and have one nontrivial saturated hereditary subset (the subset consisting of the first vertex). Thus we are in the situation of Corollary 4.4, with $E_{\mathrm{reg}}^{0}=\left\{v_{2}, v_{3}, v_{4}\right\}$ and $E_{\mathrm{reg}}^{0}=H=\left\{v_{1}\right\}$. Hence the adjacency matrix has the block form

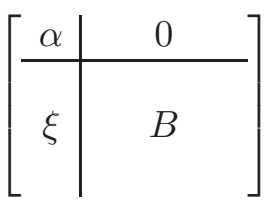

and the six-term exact sequence is

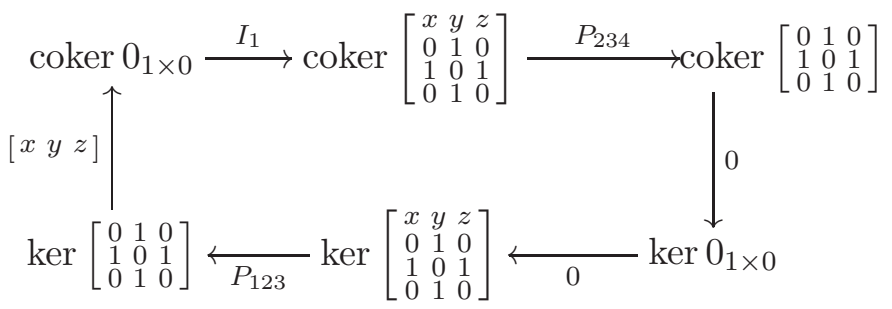

which simplifies to

$$
0 \longrightarrow \mathbb{Z} \stackrel{x-z}{\longrightarrow} \mathbb{Z} \longrightarrow \mathbb{Z} /(x-z) \oplus \mathbb{Z} \longrightarrow \mathbb{Z} \longrightarrow 0
$$

when $x \neq z$ and to

$$
0 \longrightarrow \mathbb{Z}=\mathbb{Z} \stackrel{0}{\longrightarrow} \mathbb{Z} \longrightarrow \mathbb{Z} \oplus \mathbb{Z} \longrightarrow \mathbb{Z} \longrightarrow 0
$$

when $z=x$.

The $K_{0}$-group of the ideal is canonically ordered, and the order of the $K_{0}$-group of the quotient is trivial, irrespective of $x, y, z$. We may hence apply [7] to prove that $C^{*}\left(E_{x, y, z}\right) \otimes \mathbb{K} \simeq C^{*}\left(E_{x^{\prime}, y^{\prime}, z^{\prime}}\right) \otimes \mathbb{K}$ precisely when $x-z= \pm\left(x^{\prime}-z^{\prime}\right)$.

Example 4.6. Consider the class of graphs $F_{y, z}$ given by the adjacency matrix

$$
\left[\begin{array}{ccc}
0 & 0 & 0 \\
y & 3 & 1 \\
\infty & z & 3
\end{array}\right]
$$

where $y, z \in \mathbb{N}$. These graphs all satisfy Condition $(\mathrm{K})$ and have one nontrivial saturated hereditary subset $\left\{v_{1}\right\}$ for which $\left\{v_{3}\right\}$ is breaking. We furthermore have that $E_{\text {reg }}^{0}=\left\{v_{2}\right\}$ and $E_{\text {sing }}^{0}=\left\{v_{1}, v_{3}\right\}$. If we consider the ideal $J_{\left(\left\{v_{1}\right\},\left\{v_{3}\right\}\right)}$, then the adjacency matrix has the block form

$$
\left[\begin{array}{c|c|c}
* & 0 & 0 \\
\hline \xi & B & \eta \\
\hline * & \Gamma & Z
\end{array}\right]
$$


which gives

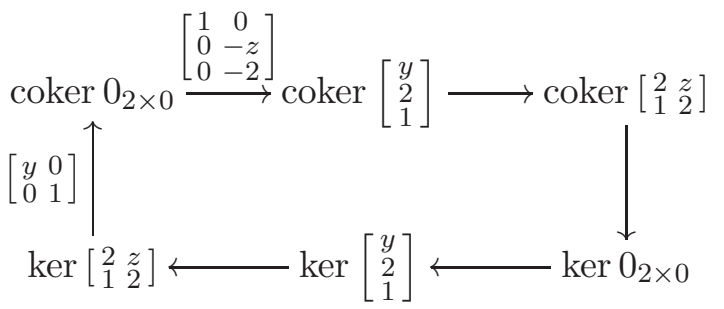

simplifying to

$$
0 \longrightarrow \mathbb{Z}^{2} \stackrel{\left[\begin{array}{cc}
-1 & -2 y \\
0 & 4-z
\end{array}\right]}{\longrightarrow} \mathbb{Z}^{2} \longrightarrow \mathbb{Z}_{z-4} \longrightarrow 0
$$

when $z \neq 4$ and to

$$
0 \longrightarrow \mathbb{Z} \stackrel{\left[\begin{array}{c}
-2 y \\
1
\end{array}\right]}{\longrightarrow} \mathbb{Z}^{2} \stackrel{\left[\begin{array}{cc}
-1 & -2 y \\
0 & 0
\end{array}\right]}{\longrightarrow} \mathbb{Z}^{2} \longrightarrow \mathbb{Z} \longrightarrow 0
$$

when $z=4$.

In both cases, the $K_{0}$-group of the ideal is ordered by

$$
\left\{\left(x_{1}, x_{3}\right): x_{3}>1 \text { or }\left[x_{3}=0, x_{1} \geq 0\right]\right\},
$$

having only the trivial automorphism, so the computations combine with [7, Theorem 4.7] to show that $C^{*}\left(F_{y, z}\right) \otimes \mathbb{K} \simeq C^{*}\left(F_{y^{\prime}, z^{\prime}}\right) \otimes \mathbb{K}$ precisely when $4-z= \pm\left(4-z^{\prime}\right)$ and $y-y^{\prime} \in(4-z) \mathbb{Z}$.

\section{PROOF OF MAIN RESUlT}

The isomorphism of the two six-term exact sequences in Theorem 4.1 is given by the six group isomorphisms $\chi_{0}^{\prime}, \chi_{0}, \chi_{0}^{\prime \prime}, \chi_{1}^{\prime}, \chi_{1}, \chi_{1}^{\prime \prime}$ defined as follows. If we let $E_{(H, S)}$ be the subgraph of $E$ with vertices $H \cup S$ and edges $s^{-1}(H) \cup$ $\left(s^{-1}(S) \cap r^{-1}(H)\right)$, then the graph $C^{*}$-algebra $C^{*}\left(E_{(H, S)}\right)$ is isomorphic to a full corner of $J_{(H, S)}$ via an embedding $\phi: C^{*}\left(E_{(H, S)}\right) \rightarrow J_{(H, S)}$ with $\phi\left(p_{v}\right)=$ $p_{v}$ for $v \in H, \phi\left(p_{v_{0}}\right)=p_{v_{0}}^{H}$ for $v_{0} \in S$ and $\phi\left(s_{e}\right)=s_{e}$ for $e \in E_{(H, S)}^{1}$ (cf. [2]). Notice that $\left(E_{(H, S)}\right)_{\mathrm{reg}}^{0}=E_{\mathrm{reg}}^{0} \cap H$ and that $\left(E_{(H, S)}\right)_{\text {sing }}^{0}=\left(E_{\text {sing }}^{0} \cap H\right) \cup S$. It follows (for example by [12, Proposition 1.2]) that $\phi$ induces an isomorphism $\phi_{*}: K_{*}\left(C^{*}\left(E_{(H, S)}\right)\right) \rightarrow K_{*}\left(J_{(H, S)}\right)$. Thus if we let $\chi_{*}^{E_{(H, S)}}$ denote the group isomorphisms given by Proposition 3.8 for $C^{*}\left(E_{(H, S)}\right)$, then

$$
\chi_{0}^{\prime}:=\phi_{*} \circ \chi_{0}^{E_{(H, S)}}: \text { coker }\left[\begin{array}{c}
A^{t}-I \\
\alpha^{t} \\
0
\end{array}\right] \rightarrow K_{0}\left(J_{(H, S)}\right)
$$

and

$$
\chi_{1}^{\prime}:=\phi_{*} \circ \chi_{1}^{E_{(H, S)}}: \operatorname{ker}\left[\begin{array}{c}
A^{t}-I \\
\alpha^{t} \\
0
\end{array}\right] \rightarrow K_{1}\left(J_{(H, S)}\right)
$$

are group isomorphisms. Similarly, if we let $E \backslash H$ be the subgraph of $E$ with vertices $E^{0} \backslash H$ and edges $r^{-1}\left(E^{0} \backslash H\right)$, then there is an isomorphism $\psi: C^{*}(E \backslash H, S) \rightarrow C^{*}(E) / J_{(H, S)}$ which for any $v \in E^{0} \backslash H$ maps $p_{v}$ to 
$p_{v}+J_{(H, S)}$ and for any $e \in r^{-1}\left(E^{0} \backslash H\right)$ maps $s_{e}$ to $s_{e}+J_{(H, S)}$ (cf. [11, Example 3.10]). Notice that $(E \backslash H)_{\mathrm{reg}}^{0}=E_{\mathrm{reg}}^{0} \backslash H$ and that $(E \backslash H)_{\mathrm{sing}}^{0}=$ $E_{\text {sing }}^{0} \backslash H$. Thus if we let $\chi_{*}^{(E \backslash H, S)}$ denote the group isomorphisms given by Proposition 3.8 for $C^{*}(E \backslash H, S)$, then

$$
\chi_{0}^{\prime \prime}:=\psi_{*} \circ \chi_{0}^{(E \backslash H, S)}: \text { coker }\left[\begin{array}{cc}
B^{t}-I & \Gamma^{t} \\
\beta^{t} & \gamma^{t} \\
\eta^{t} & Z^{t}-I
\end{array}\right] \rightarrow K_{0}\left(C^{*}(E) / J_{(H, S)}\right)
$$

and

$$
\chi_{1}^{\prime \prime}:=\psi_{*} \circ \chi_{1}^{(E \backslash H, S)}: \operatorname{ker}\left[\begin{array}{cc}
B^{t}-I & \Gamma^{t} \\
\beta^{t} & \gamma^{t} \\
\eta^{t} & Z^{t}-I
\end{array}\right] \rightarrow K_{1}\left(C^{*}(E) / J_{(H, S)}\right)
$$

are group isomorphisms. Finally we let $\chi_{*}$ denote the group isomorphisms given directly by Proposition 3.8 for $C^{*}(E)$.

The theorem then follows from the ensuing six claims.

Claim 5.1. $\iota_{*} \circ \chi_{0}^{\prime}=\chi_{0} \circ \widetilde{I}$.

Proof. If $v \in H$, then we have that

$$
\begin{aligned}
\chi_{0} \circ\left[\begin{array}{ccc}
I & 0 & 0 \\
0 & I & 0 \\
0 & 0 & -\Gamma^{t} \\
0 & 0 & -\gamma^{t} \\
0 & 0 & I-Z^{t}
\end{array}\right]\left(\mathbf{e}_{v}+\operatorname{im}\left[\begin{array}{c}
A^{t}-I \\
\alpha^{t} \\
0
\end{array}\right]\right) & =\chi_{0}\left(\mathbf{e}_{v}+\operatorname{im}\left[\begin{array}{cc}
A^{t}-I & X^{t} \\
\alpha^{t} & \xi^{t} \\
0 & B^{t}-I \\
0 & \beta^{t} \\
0 & \eta^{t}
\end{array}\right]\right) \\
& =\left[p_{v}\right]_{0}=\left[\iota\left(\phi\left(p_{v}\right)\right)\right]_{0} \\
& =\iota_{*} \circ \chi_{0}^{\prime}\left(\mathbf{e}_{v}+\operatorname{im}\left[\begin{array}{c}
A^{t}-I \\
\alpha^{t} \\
0
\end{array}\right]\right),
\end{aligned}
$$

and if $v_{0} \in S$, the left hand side equals

$$
\begin{aligned}
& \chi_{0}\left(\mathbf{e}_{v_{0}}-\sum_{\substack{s(e)=v_{0} \\
r(e) \notin H}} \mathbf{e}_{r(e)}+\operatorname{im}\left[\begin{array}{cc}
A^{t}-I & X^{t} \\
\alpha^{t} & \xi^{t} \\
0 & B^{t}-I \\
0 & \beta^{t} \\
0 & \eta^{t}
\end{array}\right]\right) \\
= & {\left[p_{v_{0}}\right]_{0}-\sum_{\substack{s(e)=v_{0} \\
r(e) \notin H}}\left[s_{e} s_{e}^{*}\right]_{0}=\left[\iota\left(p_{v_{0}}^{H}\right)\right]_{0}=\left[\iota\left(\phi\left(p_{v}\right)\right)\right]_{0} } \\
= & \iota_{*} \circ \chi_{0}^{\prime}\left(\mathbf{e}_{v}+\operatorname{im}\left[\begin{array}{c}
A^{t}-I \\
\alpha^{t} \\
0
\end{array}\right]\right) .
\end{aligned}
$$

Claim 5.2. $\pi_{*} \circ \chi_{0}=\chi_{0}^{\prime \prime} \circ P_{345}$.

Proof. As above, we check the claim of each class given by $\mathbf{e}_{v}$. If $v \in H$, then both sides vanish. If $v \notin H$, both sides equal $\left[p_{v}\right]_{0}$.

Claim 5.3. $\pi_{*} \circ \chi_{1}=\chi_{1}^{\prime \prime} \circ I_{1} \circ P_{2}$. 
Proof. Fix

$$
\mathbf{x}=\left[\begin{array}{l}
\mathbf{y} \\
\mathbf{z}
\end{array}\right] \in \operatorname{ker}\left[\begin{array}{cc}
A^{t}-I & X^{t} \\
\alpha^{t} & \xi^{t} \\
0 & B^{t}-I \\
0 & \beta^{t} \\
0 & \eta^{t}
\end{array}\right]
$$

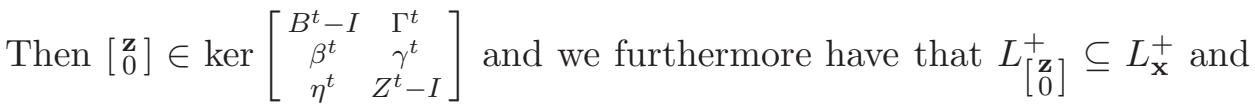
$L_{\left[\begin{array}{l}\mathbf{z} \\ 0\end{array}\right]}^{-} \subseteq L_{\mathbf{x}}^{-}$. Thus if we let $h$ be the number of elements in $L_{\mathbf{x}}^{+}\left(\right.$and in $\left.L_{\mathbf{x}}^{-}\right)$, and we let $h^{\prime}$ denote the number of elements in $L_{\left[\begin{array}{l}\mathbf{z} \\ 0\end{array}\right]}^{+}\left(\right.$and in $\left.L_{\left[\begin{array}{l}\mathbf{z} \\ 0\end{array}\right]}^{-}\right)$, then we can choose the bijections

$$
[\cdot]: L_{\mathbf{x}}^{+} \rightarrow\{1, \ldots, h\} \quad\langle\cdot\rangle: L_{\mathbf{x}}^{-} \rightarrow\{1, \ldots, h\}
$$

and

$$
[\cdot]^{\prime}: L_{\left[\begin{array}{l}
\mathbf{Z} \\
0
\end{array}\right]}^{+} \rightarrow\left\{1, \ldots, h^{\prime}\right\} \quad\langle\cdot\rangle^{\prime}: L_{\left[\begin{array}{l}
\mathbf{Z} \\
0
\end{array}\right]}^{-} \rightarrow\left\{1, \ldots, h^{\prime}\right\}
$$

such that $[\cdot]$ is an extension of $[\cdot]^{\prime}$, and $\langle\cdot\rangle$ is an extension of $\langle\cdot\rangle^{\prime}$. We then have that

$$
\begin{aligned}
\pi\left(\mathrm{V}_{\mathbf{x}}\right) & =\pi\left(\sum_{\substack{1 \leq i \leq x_{w} \\
s(e)=w}} s_{e} \mathrm{E}_{[w, i],\langle e, i\rangle}+\sum_{\substack{1 \leq i \leq-x_{w} \\
s(e)=w}} s_{e}^{*} \mathrm{E}_{[e, i],\langle w, i\rangle}\right) \\
& =\sum_{\substack{1 \leq i \leq z_{w} \\
s(e)=w}} \pi\left(s_{e}\right) \mathrm{E}_{[w, i],\langle e, i\rangle}+\sum_{\substack{1 \leq i \leq z_{w} \\
s(e)=w}} \pi\left(s_{e}^{*}\right) \mathrm{E}_{[e, i],\langle w, i\rangle} \\
& =\psi\left(\sum_{\substack{1 \leq i \leq z_{w} \\
s(e)=w}} s_{e} \mathrm{E}_{[w, i],\langle e, i\rangle}+\sum_{\substack{1 \leq i \leq-z_{w} \\
s(e)=w}} s_{e}^{*} \mathrm{E}_{[e, i],\langle w, i\rangle}\right) \\
& =\psi\left(\mathrm{V}_{\left[\begin{array}{l}
\mathbf{z}] \\
0
\end{array}\right)}\right.
\end{aligned}
$$

since $s_{e} \in J_{(H, S)}=\operatorname{ker} \pi$ when $s(e)$ (and thus $\left.r(e)\right)$ lies in $H$, and $z_{w}=x_{w}$ when $w \notin H$. A similar computation for $\mathrm{P}_{\mathbf{x}}$ shows that $\pi\left(\mathrm{P}_{\mathbf{x}}\right)=\psi\left(\mathrm{P}_{\left[\begin{array}{l}\mathbf{z} \\ 0\end{array}\right]}\right)$.

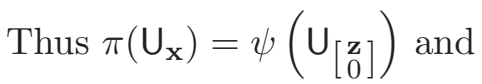

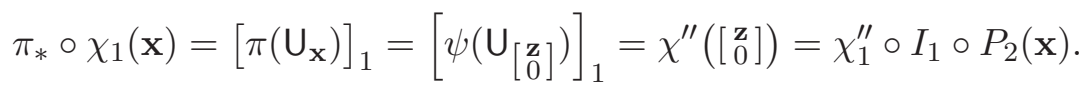

Claim 5.4. $\iota_{*} \circ \chi_{1}^{\prime}=\chi_{1} \circ I_{1}$. 
Proof. Fix $\mathbf{x} \in \operatorname{ker}\left[\begin{array}{c}A^{t}-I \\ \alpha^{t} \\ 0\end{array}\right]$. This follows like in Claim $[5.3$ by choosing the bijections

$$
[\cdot]: L_{\left[\begin{array}{l}
\mathbf{x} \\
0
\end{array}\right]}^{+} \rightarrow\{1, \ldots, h\} \quad[\cdot]: L_{\mathbf{x}}^{+} \rightarrow\{1, \ldots, h\}
$$

and

$$
\langle\cdot\rangle: L_{\left[\begin{array}{l}
\mathbf{x} \\
0
\end{array}\right]}^{-} \rightarrow\{1, \ldots, h\} \quad\langle\cdot\rangle: L_{\mathbf{x}}^{-} \rightarrow\{1, \ldots, h\}
$$

to be pairwise equal.

Claim 5.5. $\partial_{0}=0$.

Proof. It follows from Claim 5.4 that $\iota_{*}: K_{1}\left(J_{(H, S)}\right) \rightarrow K_{1}\left(C^{*}(E)\right)$ is injective. Thus $\operatorname{im}\left(\partial_{0}\right)=0$ from which it follows that $\partial_{0}=0$.

Claim 5.6. $\partial_{1} \circ \chi_{1}^{\prime \prime}=\chi_{0}^{\prime} \circ\left[\begin{array}{cc}X^{t} & 0 \\ \xi^{t} & 0 \\ 0 & I\end{array}\right]$.

Proof. Fix $\mathbf{x}=\left[\begin{array}{l}\mathbf{y} \\ \mathbf{z}\end{array}\right] \in \operatorname{ker}\left[\begin{array}{cc}B^{t}-1 & \Gamma^{t} \\ \beta^{t} & \gamma^{t} \\ \eta^{t} & Z^{t}-I\end{array}\right]$. We lift $\psi\left(\mathbf{V}_{\mathbf{x}}\right)$ and $\psi\left(\mathrm{P}_{\mathbf{x}}\right)$ to

$$
\widehat{\mathrm{V}}_{\mathbf{x}}=\sum_{\substack{1 \leq i \leq x_{w} \\ s(e)=w, r(e) \notin H}} s_{e} \mathrm{E}_{[w, i],\langle e, i\rangle}+\sum_{\substack{1 \leq i \leq-x_{w} \\ s(e)=w, r(e) \notin H}} s_{e}^{*} \mathrm{E}_{[e, i],\langle w, i\rangle}
$$

and

$$
\widehat{\mathrm{P}}_{\mathbf{x}}=\sum_{1 \leq i \leq x_{w}} p_{w} \mathrm{E}_{[w, i],[w, i]}+\sum_{\substack{1 \leq i \leq-x_{w} \\ s(e)=w, r(e)=v \\ v \notin H}} p_{v} \mathrm{E}_{[e, i],[e, i]},
$$

respectively, in $\mathrm{M}_{h}\left(C^{*}(E)\right)$. Since $\left\{s_{e}, p_{v}: e \in r^{-1}\left(E^{0} \backslash H\right), v \in E^{0} \backslash H\right\}$ is a Toeplitz-Cuntz-Krieger $(E \backslash H)$-family, it follows from Lemma 3.5 that $\widehat{V}_{x}$ is a partial isometry and that $\widehat{P}_{x} \widehat{V}_{x}=\widehat{V}_{x} \widehat{P}_{x}=\widehat{V}_{x}$. It follows that also $\widehat{U}_{\mathbf{x}}:=\widehat{V}_{\mathbf{x}}+\left(1-\widehat{P}_{\mathbf{x}}\right)$ is a partial isometry. Hence, to compute the value of the index map on $\left[\mathrm{U}_{\mathbf{x}}\right]_{1}$, we just need to compute the defect of $\widehat{U}_{\mathbf{x}}$ in $K_{0}\left(J_{(H, S)}\right)$, cf. [14, Proposition 9.2.2]. We have, using Lemma 3.4, that

$$
\begin{aligned}
& 1-\widehat{U}_{\mathrm{x}} \widehat{\mathrm{U}}_{\mathrm{x}}^{*}=\widehat{\mathrm{P}}_{\mathrm{x}}-\widehat{\mathrm{V}}_{\mathrm{x}} \widehat{\mathrm{V}}_{\mathrm{x}}^{*} \\
& =\sum_{1 \leq i \leq x_{w}}\left(p_{w}-\sum_{\substack{s(e)=w, r(e)=v \\
v \notin H}} s_{e} s_{e}^{*}\right) \mathrm{E}_{[w, i],[w, i]} \\
& =\sum_{1 \leq i \leq y_{w}}\left(\sum_{s(e)=w, r(e)=v} s_{e} s_{e}^{*}-\sum_{\substack{s(e)=w, r(e)=v \\
v \notin H}} s_{e} s_{e}^{*}\right) \mathrm{E}_{[w, i],[w, i]}
\end{aligned}
$$




$$
\begin{aligned}
& +\sum_{1 \leq i \leq z_{0}}\left(p_{v_{0}}-\sum_{s(e)=v_{0}, r(e) \notin H} s_{e} s_{e}^{*}\right) \mathrm{E}_{\left[v_{0}, i\right],\left[v_{0}, i\right]} \\
= & \sum_{1 \leq i \leq y_{w}} \sum_{\substack{s(e)=w, r(e)=v \\
v \in H}} s_{e} s_{e}^{*} \mathrm{E}_{[w, i],[w, i]}+\sum_{1 \leq i \leq z_{v_{0}}} p_{v_{0}}^{H} \mathrm{E}_{\left[v_{0}, i\right],\left[v_{0}, i\right]} .
\end{aligned}
$$

Passing to the $K_{0}$-group and using that $s_{e} \in C^{*}\left(E_{H}\right)$, we get

$$
\begin{aligned}
{\left[1-\widehat{\mathrm{U}}_{\mathbf{x}} \widehat{\mathrm{U}}_{\mathbf{x}}^{*}\right]_{0} } & =\sum_{1 \leq i \leq y_{w}} \sum_{\substack{s(e)=w, r(e)=v \\
v \in H}}\left[s_{e} s_{e}^{*}\right]_{0}+\sum_{1 \leq i \leq z_{v_{0}}}\left[p_{v_{0}}^{H}\right]_{0} \\
& =\sum_{0<y_{w}} y_{w} \sum_{\substack{s(e)=w, r(e)=v \\
v \in H}}\left[s_{e}^{*} s_{e}\right]_{0}+\sum_{0<z_{v_{0}}} z_{v_{0}}\left[p_{v_{0}}^{H}\right]_{0} \\
& =\sum_{0<y_{w}} y_{w} \sum_{\substack{s(e)=w, r(e)=v \\
v \in H}}\left[p_{v}\right]_{0}+\sum_{0<z_{v_{0}}} z_{v_{0}}\left[p_{v_{0}}^{H}\right]_{0} .
\end{aligned}
$$

The computation for $1-\widehat{\mathrm{U}}_{\mathbf{x}}^{*} \widehat{\mathrm{U}}_{\mathbf{x}}$ is similar, and we get

$$
\left[1-\widehat{\mathrm{U}}_{\mathbf{x}} \widehat{\mathrm{U}}_{\mathbf{x}}^{*}\right]_{0}-\left[1-\widehat{\mathrm{U}}_{\mathbf{x}}^{*} \widehat{\mathrm{U}}_{\mathbf{x}}\right]_{0}=\sum_{0 \neq y_{w}} y_{w} \sum_{\substack{s(e)=w, r(e)=v \\ v \in H}}\left[p_{v}\right]_{0}+\sum_{z_{v_{0}} \neq 0} z_{v_{0}}\left[p_{v_{0}}^{H}\right]_{0} .
$$

By comparison,

$$
\begin{aligned}
& \chi_{0}^{\prime}\left(\left[\begin{array}{cc}
X^{t} & 0 \\
\xi^{t} & 0 \\
0 & I
\end{array}\right] \mathbf{x}\right)=\chi_{0}^{\prime}\left(\left[\begin{array}{c}
X^{t} \mathbf{y} \\
\xi^{t} \mathbf{y} \\
\mathbf{z}
\end{array}\right]+\operatorname{im}\left[\begin{array}{c}
A^{t}-1 \\
\alpha^{t} \\
0
\end{array}\right]\right) \\
& =\sum_{y_{w} \neq 0} y_{w}\left(\sum_{v \in E_{\mathrm{reg}}^{0} \cap H} X_{v, w}\left[p_{v}\right]_{0}+\sum_{v \in E_{\mathrm{sing}}^{0} \cap H} \xi_{v, w}\left[p_{v}\right]_{0}\right) \\
& +\sum_{z_{v_{0}} \neq 0} z_{v_{0}}\left[p_{v_{0}}^{H}\right]_{0} \\
& =\sum_{x_{w} \neq 0} y_{w}\left(\sum_{\substack{s(e)=w, r(e)=v \\
v \in E_{\mathrm{reg}}^{0} \cap H}}\left[p_{v}\right]_{0}+\sum_{\substack{s(e)=w, r(e)=v \\
v \in E_{\mathrm{sing}}^{0} \cap H}}\left[p_{v}\right]_{0}\right) \\
& +\sum_{z_{v_{0}} \neq 0} z_{v_{0}}\left[p_{v_{0}}^{H}\right]_{0} \\
& =\sum_{x_{w} \neq 0} y_{w} \sum_{\substack{s(e)=w, r(e)=v \\
v \in H}}\left[p_{v}\right]_{0}+\sum_{z_{v_{0}} \neq 0} z_{v_{0}}\left[p_{v_{0}}^{H}\right]_{0},
\end{aligned}
$$

completing the proof. 


\section{REFERENCES}

[1] P. Ara, M. A. Moreno, and E. Pardo. Nonstable $K$-theory for graph algebras. Algebr. Represent. Theory, 10(2):157-178, 2007.

[2] T. Bates, D. Pask, I. Raeburn, and W. Szymanski. $C^{*}$-algebras of row-finite graphs. New York J. Math., 6:307-324, 2000.

[3] L.G. Brown and G.K. Pedersen. $C^{*}$-algebras of real rank zero. J. Funct. Anal., 99:131149, 1991.

[4] J. Cuntz. On the homotopy groups of the space of endomorphisms of a $C^{*}$-algebra (with applications to topological Markov chains). In Operator algebras and group representations, Vol. I (Neptun, 1980), volume 17 of Monogr. Stud. Math., pages 124-137. Pitman, Boston, MA, 1984.

[5] D. Drinen and M. Tomforde. Computing $K$-theory and Ext for graph $C^{*}$-algebras. Illinois J. Math., 46:81-91, 2002.

[6] S. Eilers, G. Restorff, and E. Ruiz. Classifying $C^{*}$-algebras with both finite and infinite subquotients. Preprint, arXiv:0801.0324v3, 2010.

[7] S. Eilers and M. Tomforde. On the classification of nonsimple graph algebras. Math. Ann., 346:393-418, 2010.

[8] N.J. Fowler and I. Raeburn. The Toeplitz algebra of a Hilbert bimodule. Indiana Univ. Math. J., 48(1):155-181, 1999.

[9] J.A Jeong. Real rank of $C^{*}$-algebras associated with graphs. J. Aust. Math. Soc., 77(1):141-147, 2004.

[10] T. Katsura. On $C^{*}$-algebras associated with $C^{*}$-correspondences. J. Funct. Anal., 217(2):366-401, 2004.

[11] P.S. Muhly and M. Tomforde. Adding tails to $C^{*}$-correspondences. Doc. Math., 9:79106, 2004.

[12] W. Paschke. $K$-theory for actions of the circle group on $C^{*}$-algebras. J. Operator Theory, 6(1):125-133, 1981.

[13] M. Rørdam. Classification of Cuntz-Krieger algebras. K-Theory, 9(1):31-58, 1995.

[14] M. Rørdam, F. Larsen, and N. Laustsen. An introduction to K-theory for $C^{*}$-algebras, volume 49 of London Mathematical Society Student Texts. Cambridge University Press, Cambridge, 2000.

[15] M. Tomforde. The ordered $K_{0}$-group of a graph $C^{*}$-algebra. C. R. Math. Acad. Sci. Soc. R. Can., 25(1):19-25, 2003.

Department of Mathematical Sciences, Norwegian University of Science And Technology, NO-7491 Trondheim, Norway

E-mail address: tokemeie@math.ntnu.no

Department for Mathematical Sciences, University of Copenhagen, Universitetsparken 5, DK-2100 Copenhagen $\varnothing$, Denmark

E-mail address: eilers@math.ku.dk

Department of Mathematics, University of Houston, Houston, TX 772043008, USA

E-mail address: tomforde@math.uh.edu 